\title{
ATOMIC SPACES AND SPECTRA
}

\author{
by J. F. ADAMS* and N. J. KUHN
}

(Received 28th July 1988)

1. The subject-matter of this paper is in some sense known; but we will try to organise, explain and reprove it, and to give examples.

In essence, a space or spectrum $X$ is "atomic" if a map $f: X \rightarrow X$ may be proved to be an equivalence by a simple, computable test applied in one dimension; this goes back to [4] (published as [5]) and first appeared in print in [12]. That it is useful to prove $X$ atomic and then apply the fact has been amply shown, beginning with [3].

This notion is related to two others. Unique factorisation results for spaces and spectra have been considered in $[6,9,14]$. Here one needs the notion of an "irreducible" or "indecomposable" object $X$, and a slightly stronger notion of "prime".

We first show that the case of "spaces" and the case of "spectra" can be considered together, by concentrating on the fact that the hom-set $[X, X]$ is (under suitable assumptions) a profinite monoid. In this case we show that the "weaker" condition implies the "stronger", as follows.

(a) If $X$ is indecomposable then its hom-set $[X, X]$ is "good", and

(b) if $[X, X]$ is "good" then $X$ is both "atomic" and "prime".

We give some illustrative examples, including some which arise "in nature" as stable summands of classifying spaces $B G$. We conclude with the proofs.

Related results have been obtained by $\mathbf{M}$. C. Crabb and J. R. Hubbuck; we are grateful to them for letters, and also to F. R. Cohen and F. P. Peterson.

2. First we unify the two cases to be considered.

Proposition 2.1. The hom-set $[X, X]$ is a profinite monoid with zero in both the following cases.

(2.2) $X$ is a p-complete $C W$-complex of finite type and $[X, X]$ means homotopy classes of pointed maps.

(2.3) $X$ is a p-complete spectrum of finite type and $[X, X]$ means maps in the homotopy category of spectra.

We will comment in Section 3.

*The Society is saddened by the sudden death on 7 January 1989 of Professor J. F. Adams, F.R.S. 
Proposition 2.4. Suppose $M$ is a profinite monoid with zero. Then either

(a) $M$ contains a non-trivial idempotent, or

(b) $M$ is "good" in that the sense that each $f \in M$ is either invertible or topologically nilpotent.

In (a), an idempotent is "non-trivial" if it is neither 0 nor 1.

In (b), " $f$ is topologically nilpotent" means that as $n \rightarrow \infty$, so $f^{n} \rightarrow 0$ in the profinite topology on $M$.

The proof of (2.4), which is elementary, will be given in Section 3.

When $[X, X]$ contains a non-trivial idempotent, $X$ is "reducible" or "decomposable". For spaces this means that $X$ has a non-trivial retract; that is, there is a diagram

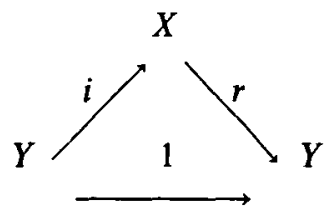

in which $Y$ is not contractible and $i, r$ are not equivalences. For spectra we can go on to infer a non-trivial decomposition as a wedge-sum, $X \simeq Y \vee Z$.

If $X$ is "irreducible" or "indecomposable" then the possibility of a non-trivial idempotent is excluded, and we conclude that $[X, X]$ is "good".

Thus (2.1) plus (2.4) is an analogue, for homotopy-theorists, of a well-known algebraic result: under suitable finiteness conditions, if an $R$-module $X$ is indecomposable, then every map $f: X \rightarrow X$ is either invertible or nilpotent. We were surprised to find that such a result survives in a context with no addition.

We will sketch the argument that if $[X, X]$ is good, then $X$ is atomic. In the applications, a simple computable test will dismiss the possibility that $f$ is topologically nilpotent. For example, suppose we choose any dimension $n$ where $H_{n}\left(X ; F_{p}\right) \neq 0$. If $f$ is an equivalence, then $f_{*}: H_{n}\left(X ; F_{p}\right) \rightarrow H_{n}\left(X ; F_{p}\right)$ must be iso. But conversely, if $f_{*}: H_{n}\left(X ; F_{p}\right) \rightarrow H_{n}\left(X ; F_{p}\right)$ is iso, then it cannot be nilpotent, so $f$ cannot be topologically nilpotent, and $f$ must be an equivalence (assuming $[X, X]$ is good). We conclude that $X$ is atomic.

Of course, many functors other than $H_{n}\left(-; F_{p}\right)$ would serve as well.

We will sketch the argument that if $[X, X]$ is good, then $X$ is "prime".

If $f$ and $g$ are both topologically nilpotent, then the equation

$$
f+g=1 \neq 0
$$

cannot hold even after passing to a finite quotient $M_{\alpha}$ of $M$ and embedding $M_{\alpha}$ in a ring $R$ (where we can add). In fact, in the finite quotient $M_{\alpha}$ we would have $f^{m}=0$, $g^{n}=0$; in $R, f$ would commute with $1-f=g$; so we would have $(f+g)^{m+n-1}=0$.

We now assume that " $X$ divides $Y Z$ ". For spectra this means that we assume given a retraction 


$$
X \rightarrow Y \vee Z \rightarrow X .
$$

We take $f, g$ to be the composites

$$
\begin{aligned}
& X \rightarrow Y \rightarrow X \\
& X \rightarrow Z \rightarrow X .
\end{aligned}
$$

We then have $f+g=1$ in $[X, X]$; assuming $[X, X]$ is good, we deduce that either $f$ or $g$ is an equivalence, and $X$ is a retract either of $Y$ or of $Z$. That is, "if $X$ divides $Y Z$, then $X$ divides either $Y$ or $Z$ ".

For spaces, $Y \vee Z$ should become $Y \times Z$. We cannot argue in quite the same way because we cannot add in $[X, X]$; but we can obtain the equation $f+g=1$ after passing to a suitable ring $R=\operatorname{End} h(X)$, where $h$ is a suitable functor $\pi_{r}(-) \otimes F_{p}$ or $Q H^{r}\left(-; F_{p}\right)$ with $h(X) \neq 0$.

We turn to the examples.

Example 2.5 There is a $p$-local spectrum $X$ of finite type which is indecomposable (but becomes decomposable on completion) and for which $[X, X]$ is not good.

This justifies the assumption of $p$-completeness above. The construction will be given in Section 4.

In the case of spectra, $[X, X]$ is a profinite ring $R$. When $R$ is good it is local: the topologically-nilpotent elements make up the unique maximal ideal $\operatorname{rad}(R)$. (Given the indications above, the proof may be left to the reader; the result is due to [9].)

Remark 2.6. In this case the quotient $R / \operatorname{rad}(R)$ is a finite field.

The proof is easy, but this too is postponed to Section 4 .

This raises the question, which finite fields occur as $R / \operatorname{rad}(R)$. Here we present two examples; one involves infinite spectra which "arise in nature", and the other involves finite spectra constructed by hand.

Example 2.7. Each finite field arises as $R / \operatorname{rad}(R)$ for a suitable $X$ which is an indecomposable stable summand of a classifying space $B G$. Indeed, if the field is of characteristic $p$, then $G$ can be a $p$-group.

For simplicity we now take $p=2$.

Example 2.8. Each finite field of characteristic 2 arises as $R / \operatorname{rad}(R)$ for a suitable $X$ which is a finite spectrum

The constructions will be given in Section 4 .

3. We begin by commenting on (2.1).

The cases of spaces, (2.2), is presumably known; but we sketch a proof avoiding certain difficulties.

First we set up some finite quotients of the monoid $N=[X, X]$. Let $X_{\alpha}$ be a space 
whose homotopy groups $\pi_{r}\left(X_{\alpha}\right)$ are finite $p$-groups, and zero except for a finite number of $r$. Then $\left[X, X_{\alpha}\right]$ is a finite set and $M=[X, X]$ acts on it from the right; let $M_{\alpha}$ be the image of $M$ in the monoid End $\left(\left[X, X_{a}\right]\right)$. Then $M_{a}$ is a finite monoid; and the map $M \rightarrow M_{\alpha}$ is continuous because the map $m \mapsto i_{\alpha} m:[X, X] \rightarrow\left[X, X_{\alpha}\right]$ is continuous for each of the finitely many $i_{\alpha}$ in $\left[X, X_{\alpha}\right]$.

Secondly we note that the map $M \rightarrow \prod_{\alpha} M_{\alpha}$ is mono. In fact, according to Sullivan [13] we can arrange an isomorphism

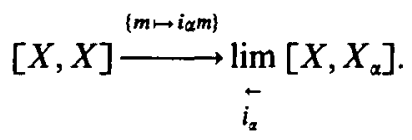

Thus $i_{\alpha} m^{\prime}=i_{\alpha} m^{\prime \prime}$ for all $i_{\alpha}$ implies $m^{\prime}=m^{\prime \prime}$.

Thirdly we order the $M_{\alpha}$ by considering diagrams

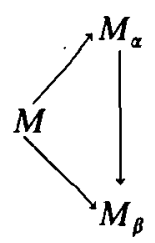

(without requiring any relation between the spaces $X^{\alpha}, X_{\beta}$ ). We show that the map.

$$
M \rightarrow \lim M_{\alpha}
$$

is an epi by a standard compactness argument.

This completes the sketch proof that $[X, X]$ is a profinite monoid.

The case of spectra, (2.3), is due to [9, Proposition 4, p. 155\}.

We turn to the proof of (2.4).

Suppose given $f \in M$. Let $M_{\alpha}$ be a finite quotient of $M$. We show first that some power $f^{n}$ of $f$ (with $n \geqq 1$ ) becomes idempotent in $M_{\alpha}$.

In fact, if infinitely many powers $f^{n}$ lie in the same finite set $M_{\infty}$ then two of them must be equal, say $f^{a}=f^{a+b}$ for some $a \geqq 1, b \geqq 1$. Applying $f$ and iterating we get $f^{c}=f^{c+b d}$ for $c \geqq a$. Taking $b d \geqq a$ we get $f^{b d}=f^{2 b d}$ is idempotent in $M_{\alpha}$.

Next let $F$ be $\left\{f^{n} \mid n \geqq 1\right\}$, the set of powers of $f$, and let $\bar{F}$ be the closure of $F$ in $M$. We show that $\bar{F}$ contains an idempotent (possibly 0 or 1). In fact, for each finite quotient $M_{\alpha}$ of $M$, let $E_{\alpha}$ be the set of elements in $\bar{F}$ which map to idempotents in $M_{\alpha}$. Then $E_{\alpha}$ is closed and non-empty (for by the last paragraph it contains some power of $f)$. Indeed, the sets $E_{\alpha}$ have the finite intersection property, for any finite intersection $E_{\alpha} \cap E_{\beta} \cap \ldots \cap E_{\delta}$ contains another $E_{\varepsilon}$ (consider the pull-back of $M_{\alpha}, M_{\beta}, \ldots, M_{\delta}$ ). $M$ is compact, so there is an element common to all the $E_{\alpha}$, i.e. an idempotent in $\bar{F}$.

It is possible that the idempotent in $\bar{F}$ is 1 ; in this case we will show that $f$ is invertible. In fact, assume $1 \in \bar{F}$; then in each finite quotient $M_{\alpha}$ of $M$ we have $1=f^{n}$ for 
some $n \geqq 1$, so $f$ has an inverse $1=f^{n-1}$ in $M_{x}$. This inverse is unique, and these inverse elements give an element of $\lim _{\leftarrow} M_{\alpha}$, providing an inverse for $f$ in $M$.

It is possible that the idempotent in $\bar{F}$ is 0 ; in this case we will show that $f$ is topologically nilpotent. In fact, assume $0 \in \bar{F}$, and let $M_{a}$ be a finite quotient of $M$. Then some $f^{n}$ maps to 0 in $M_{x}$, hence $f^{r}$ maps to 0 in $M_{\alpha}$ for $r \geqq n=n(\alpha)$. Thus $f^{r} \rightarrow 0$ in the profinite topology.

This completes the proof of (2.4).

4. We begin with (2.5).

Our spectrum $X$ will have $H_{0}(X)=Z_{(p)} \oplus Z_{(p)}$, so that $\operatorname{End}\left(H_{0}(X)\right)$ is a ring of $2 \times 2$ matrices. We will construct $X$ so that the image of

$$
[X, X] \rightarrow \operatorname{End}\left(H_{0}(X)\right)
$$

is a ring

$$
\frac{Z_{(p)}[A]}{\left(A^{2}-A+p\right)}
$$

The proposed minimum polynomial $x^{2}-x+p$ has no real roots (because $b^{2}-4 a c<0$ ), and a fortiori no roots in $Z_{(p)}$. It does have $p$-adic roots $\alpha_{0}, \alpha_{1}$ congruent to $0,1 \bmod p$ (e.g. by Hensel's Lemma). A convenient matrix with this minimum polynomial is

$$
A=\left[\begin{array}{rr}
0 & 1 \\
-p & 1
\end{array}\right]
$$

this has $\left(1, \alpha_{0}\right)^{T},\left(1, \alpha_{1}\right)^{T}$ as eigenvectors with eigenvalues $\alpha_{0}, \alpha_{1}$.

Next we need a sequence of elements $g_{i} \in \pi_{n_{i}-1}^{s}\left(S^{0}\right), i=1,2, \ldots$, such that $g_{i}$ has order $p^{i}$ in $\pi_{*}^{S}\left(S^{0}\right)$ and still has order $p^{i}$ in $\pi_{*}^{S}\left(S^{0}\right)^{*} / I_{i}$, where $I_{i}$ is the ideal generated by the $g_{j}$ with $j \neq i$. These conditions can easily be satisfied by suitable elements in the image of the $J$-homomorphism.

We now take

$$
X=\left(S^{0} \vee S^{0}\right) \cup\left(\bigcup_{i} e^{n_{i}}\right),
$$

where we work localised at $p$ but omit the notation for it, and where the attaching map for $e^{n_{i}}$ has components $\left(g_{i}, \alpha_{i} g_{i}\right)$. (Here $\alpha_{i}$ means $\alpha_{0}$ or $\alpha_{1}$ according as $i$ is even or odd.) This has the following effect. A self-map $f$ of $S^{0} \vee S^{0}$, given by a matrix $B$, extends over $e^{n_{i}}$, with degree $d_{i}$ on $e^{n_{i}}$, if and only if $\left(1, \alpha_{i}\right)^{T}$ is an eigenvector for $B \bmod p^{i}$, with eigenvalue $d_{i} \bmod p^{i}$; it extends over $X$ if and only if this condition holds for all $i$, that is, if and only if $\left(1, \alpha_{0}\right)^{T}$ and $\left(1, \alpha_{1}\right)^{T}$ are $p$-adic eigenvectors for $B$. By construction $A$ satisfies this condition, so $A$ comes from a map $X \rightarrow X$. Conversely, suppose $B$ satisfies 
it; then $B$ is a $p$-adic linear combination of $I$ and $A$; here the coefficients of $I, A$ are the entries $B_{11}, B_{12}$ in $B$, so they lie in $Z_{(p)}$. This proves that the image of

$$
[X, X] \rightarrow \operatorname{End}\left(H_{0}(X)\right)
$$

is

$$
\frac{Z_{(p)}[A]}{\left(A^{2}-A+p\right)}
$$

We show that $X$ is indecomposable (over $Z_{(p)}$ ). In fact,

$$
\frac{Z_{(p)}[A]}{\left(A^{2}-A+p\right)}
$$

is in integral domain; so for any idempotent $e \in[X, X]$, either $e$ or $1-e$ maps to 0 in End $H_{0}(X)$, and the other maps to 1 ; the one which maps to 1 maps each cell $e^{n_{1}}$ with degree congruent to $1 \bmod p^{i}$, and must be an equivalence.

We show that $[X, X]$ is not good. In fact, the map which realises $A$ is neither an equivalence nor topologically nilpotent, for on $H_{0}\left(X ; F_{p}\right)$ it induces an idempotent of rank 1.

Proof of (2.6). Consider the quotient map $q$ from $R$ to a finite quotient ring $R_{a} \neq 0$. Under $q$ invertible elements map to invertible elements, and topologically nilpotent elements map to nilpotent ones; thus $q^{-1}\left(\operatorname{rad}\left(R_{\alpha}\right)\right)=\operatorname{rad}(R)$ and

$$
R / \operatorname{rad}(R) \cong R_{\alpha} / \operatorname{rad}\left(R_{\alpha}\right)
$$

So $R / \operatorname{rad} R$ is finite; being a finite division algebra, it must be a finite field.

We turn to (2.7). Here we need some hold on the ring of stable maps $\left\{B G_{+}, B G_{+}\right\}$.

Lemma 4.1. Let $G$ be a finite p-group. Then the group ring $F_{p}[\mathrm{Out}(G)]$ is a quotient of the ring $\left\{B G_{+}, B G_{+}\right\}$.

The obvious map is in the direction

$$
Z[\mathrm{Out}(G)] \rightarrow\left\{B G_{+}, B G_{+}\right\} ;
$$

but we definitely need a quotient of $\left\{B G_{+}, B G_{+}\right\}$.

Sketch proof. The ring $\left\{B G_{+}, B G_{+}\right\}$is known $[11$, p. 397 , Corollary $2.3 ; 10$, p. 128 , Corollary 15] as a consequence of the Segal conjecture. In particular, we have 


$$
F_{p} \otimes\left\{B G_{+}, B G_{+}\right\} \cong F_{p} \otimes A(G, G)
$$

where $A(G, G)$ is a ring that plays the same role here that the Burnside ring $A(G)$ does in studying $\left\{B G_{+}, S^{0}\right\}[1,10]$. (In [11] the present $A(G, G)$ is written $F(G, G)$.) As a $Z$-module, $A(G, G)$ is free, with a base of elements which may be written $\theta_{*} i^{*}$. Here $i$ runs over the inclusions of subgroups $i: H \rightarrow G$, and $i^{*}$ corresponds to the transfer map $\operatorname{Tr}: B G_{+} \rightarrow B H_{+} ; \theta$ runs over homomorphisms $\theta: H \rightarrow G$, and $\theta_{*}$ corresponds to the induced map $B \theta_{+}: B H_{+} \rightarrow B G_{+}[1$, Section $9 ; 7$, p. 433]. If $\theta$ is epi, then we must have $H=G$ and $i=1$, and $\theta$ must be iso. Let $I$ be the $Z$-submodule of $A(G, G)$ generated by the remaining elements $\theta_{*} i^{*}$, in which $\theta$ is not epi. We claim $I$ is an ideal.

Consider a product $\theta_{*} i^{*} \phi_{*} j^{*}$, and assume first that $\theta$ is not epi. The product $i^{*} \phi_{*} j^{*}$ can be reduced to a sum of terms $\sum_{\alpha}\left(\psi_{\alpha}\right)_{*} k^{*}$, so we obtain a sum of terms $\left(\theta \psi_{\alpha}\right)_{*} k^{*}$ in which $\theta \psi_{\alpha}$ is not epi.

Assume secondly that $\phi$ is not epi. By the last paragraph it is sufficient to consider the case in which $\theta$ is iso and $i=1$; but then we get $(\theta \phi)_{*} j^{*}$, in which $\theta \phi$ is not epi. Thus $I$ is an ideal.

We now see that the quotient ring $A(G, G) / I$ is $Z[$ Out $(G)]$. (Two automorphisms $\theta$ of $G$ give the same basis element in $A(G, G)$ if and only if they differ by conjugation in $G$ ). Thus

$$
A(G, G) /(I+(p)) \cong F_{p}[\operatorname{Out}(G)]
$$

and this proves the lemma.

Lemma 4.2. The finite field $F_{q}$, where $q=p^{n}$, may be obtained as a quotient of the ring $\left\{B G_{+}, B G_{+}\right\}$for a suitable p-group $G$.

Proof. By a theorem of Bryant and Kovacs [2,8, p. 403, Theorem 13.5] there is a p-group $G$ whose abelianisation $G /[G, G]$ is the additive group $(Z / p)^{n}$ of $F_{q}$ and whose automorphism group Aut $G$ acts on $G /[G, G]$ as the multiplicative group $F_{q}^{\times}$of $F_{q}$. Clearly this action factors through $\operatorname{Out}(G)$, so we get epimorphisms

$$
F_{p}[\operatorname{Out}(G)] \rightarrow F_{p}\left[F_{q}^{\times}\right] \rightarrow F_{q}
$$

Using (4.1), we get a map of rings from $\left\{B G_{+}, B G_{+}\right\}$onto $F_{q}$.

(2.7) now follows. If we take a complete decomposition of 1 into orthogonal idempotents in $\left\{B G_{+}, B G_{+}\right\}$, then just one of the idempotents maps to 1 in $F_{q}$; if $X$ is the corresponding summand of $B G_{+}$, then $\{X, X\}$ maps onto $F_{q}$ and $R / \operatorname{rad}(R) \cong F_{q}$, as in the proof of $(2.6)$.

We turn to (2.8). In order to realise the finite field $F_{2 q}$, we begin with $W=\bigvee_{1}^{q} S^{0}$. (We work completed, but for simplicity we omit the notation for it.) We next form

$$
X=W \cup_{f} C S^{8} W
$$

where the attaching map $f$ has to be described. For any $w \in \pi_{0}(W), f$ is to carry $S^{8} w$ to 


$$
w \cdot \bar{v}+\phi(w) \cdot \varepsilon
$$

here $\bar{v}, \varepsilon$ are the two generators for $\pi_{8}^{S}\left(S^{0}\right)=Z_{2} \oplus Z_{2}$, and $\phi$ has to be described. Since the result depends only on $\phi(w) \bmod 2$, we may interpret $\phi$ as an endomorphism of

$$
V=\pi_{0}(W) \otimes F_{2}=H_{0}\left(W ; F_{2}\right)=H_{0}\left(X ; F_{2}\right) .
$$

We take $\phi: V \rightarrow V$ to be some linear map whose minimum polynomial is an irreducible polynomial $P$ of degree $q$ over $F_{2}$.

An endomorphism of $H_{*}\left(X ; F_{2}\right)$ is now given by a linear map $\lambda: V \rightarrow V$ in degree 0 and a linear map $\mu: V \rightarrow V$ in degree 9 . Such a pair $(\lambda, \mu)$ is induced by a map $g: X \rightarrow X$ if and only if it commutes with the boundary map, that is

$$
\lambda w \cdot \bar{v}+\lambda \phi w \cdot \varepsilon=\mu w \cdot \bar{v}+\phi \mu w \cdot \varepsilon .
$$

Equivalently, $\lambda=\mu$ and $\lambda \phi=\phi \lambda$, that is, $\lambda$ commutes with $\phi$.

Multiplication by $\phi$ gives $V$ the structure of a module over $F_{2}[\phi] / P \cong F_{2 q}$; this structure is of course a 1 -dimensional vector space over $F_{2 q}$. The possible maps $\lambda$ are the endomorphisms of this structure, i.e. multiplication by the elements of $F_{29}$. This shows that the image of

$$
R=[X, X] \rightarrow \operatorname{End}(V)
$$

is $F_{2 q}$.

It is now clear that $X$ is indecomposable; and $R / \operatorname{rad}(R) \cong F_{24}$, as in the proof of (2.6).

Acknowledgement. The second author gratefully acknowledges the support of the Sloan Foundation, the S.E.R.C. and the N.S.F.

\section{REFERENCES}

1. J. F. Adams, J. H. Gunawardena and H. Miller, The Segal conjecture for elementary abelian p-groups, Topology 24 (1985), 435-460.

2. R. M. Bryant and L. G. Kovacs, Lie representations and groups of prime power order, $J$. London Math. Soc. 17 (1978), 415-421.

3. F. R. Cohen and M.E. Mahowald, A remark on the self-maps of $\Omega^{2} S^{2 n+1}$, Indiana Univ. Math. J. 30 (1981), 583-588.

4. F. R. Cohen, J. C. Moore and J. A. Neisendorfer, Moore spaces have exponents, preprint, circa 1981 .

5. F. R. Cohen, J. C. Moore and J. A. Neisendorfer, Exponents in homotopy theory, in Algebraic Topology and Algebraic K-Theory (Annals of Mathematics Studies no. 113, Princeton University Press 1987), 3-34.

6. P. J. FREYD, Stable homotopy, in Proceedings of the Conference on Categorical Algebra, La Jolla 1965 (Springer 1966), 121-172. 
7. J. C. Harris and N. J. KuHN, Stable decompositions of classifying spaces of finite abelian p-groups, Math. Proc. Cambridge Philos. Soc. 103 (1988), 427-449.

8. B. Huppert and N. Blackburn, Finite Groups II (Springer 1982).

9. H. R. Margolis, Spectra and Steenrod Algebra (North-Holland 1983).

10. J. P. MaY, Stable maps between classifying spaces, Contemp. Math. 37 (1985), 121-129.

11. G. Nishida, Stable homotopy type of classifying spaces of finite groups, in Algebraic and Topological Theories-to the Memory of Dr Takehiko Miyata (Kinokuniya, Tokyo 1985), 391-404.

12. P. S. Selick, On the indecomposability of certain sphere-related spaces (Canadian Math. Soc. Conference Proceedings Vol. 2, Part 1, 1982), 359-372.

13. D. Sullivan, Genetics of homotopy theory and the Adams conjecture, Ann. of Math. 100 (1974), 1-79.

14. C. W. Wilkerson, Genus and cancellation, Topology 14 (1975), 29-36.

Department of Mathematics

UNIVERSITY OF VIRGINIA

Charlottesville, VA 22903

USA 